Service social

\title{
Un enjeu d'amour : des réflexions d'adolescentes sur leurs relations avec les garçons
}

\section{Marie Drolet}

Volume 37, numéro 1-2, 1988

Par-delà les barrières des sexes

URI : https://id.erudit.org/iderudit/706389ar

DOI : https://doi.org/10.7202/706389ar

Aller au sommaire du numéro

Éditeur(s)

École de service social de l'Université Laval

ISSN

1708-1734 (numérique)

Découvrir la revue

Citer cet article

Drolet, M. (1988). Un enjeu d'amour : des réflexions d'adolescentes sur leurs relations avec les garçons. Service social, 37(1-2), 137-157.

https://doi.org/10.7202/706389ar
Résumé de l'article

Cet article présente l'analyse du point de vue de dix adolescentes usagères du Centre de santé des femmes de la Mauricie sur leurs relations avec les garçons. Ce thème fut étudié par rapport à leur processus contraceptif dans le cadre d'une recherche qualitative visant à sonder l'intégration de la définition féministe du concept d'autonomie chez ces usagères. Les données d'entrevue furent recueillies auprès d'un échantillon typique en début de 1987 et traitées selon une méthode d'analyse de contenu de représentation soit : les co-occurrences associatives. Malgré une image idéalisée de leur partenaire et idyllique de leurs amours, les adolescentes s'interrogent et tentent des démarches d'autonomie, quoique de façon plutôt intuitive. 
Drolet, Marie, travailleuse sociale au

C.L.S.C. Les Forges, Trois-Rivières.

\section{Un enjeu d'amour : des réflexions d'adolescentes sur leurs relations avec les garçons}

\section{Marie Drolet *}

Depuis quelques années, la problématique de la contraception à l'adolescence attire l'attention des travailleurs sociaux. En effet, l'âge moyen de la première relation coïtale ayant baissé à 15-16 ans, le danger de grossesses indésirées s'accroît. Les difficultés de contraception s'expliquent, notamment, par le processus de maturation lié à cette étape développementale : le passage vers la pensée formelle et vers la responsabilisation individuelle. Les écrits, notamment du service de pédiatrie de l'Hôpital Sainte-Justine (Frappier, 1980; Frappier, 1982; Wilkins, 1985 ; Desrosiers, 1986) et de la Clinique des jeunes Saint-Denis (Charbonneau et al., 1984 ; Tessier, 1984, 1985 et 1986) mettent en valeur cette vision globale, bio-psycho-sociale de l'adolescence et de la sexualité à cet âge.

En outre, l'intérêt pour cette problématique s'est accentué durant l'année 1987. Les études récentes désignent les 15-19 ans comme la clientèle à risques élevés pour les maladies transmises sexuellement (MTS), spécialement pour la gonorrhée et le chlamydia (Charbonneau et al., 1984; Beaulieu, 1986; Steben, 1986 a et b; Beaulieu, 1987; Germain, 1987; Kantrowitz et al., 1987). Ils sont, depuis août 1987, le groupe-cible de la campagne de prévention, notamment du SIDA, menée par le Ministère de la santé et des services sociaux du Québec (Gagnon, 1987).

Cet article, qui soulève un thème étudié dans notre mémoire de maîtrise (Drolet, 1987), offre un apport complémentaire à ces deux facettes. Nous y discuterons, en effet, de la perception d'adolescentes sur leurs relations avec les garçons. Nous délaisserons alors la dimension biologique du processus contraceptif pour axer notre réflexion sur le psychosocial et spécialement sur le contexte où se réalisent cette sexualité et cette contraception. 


\section{Objet}

L'objet de notre recherche visait à sonder l'intégration de la définition féministe du concept d'autonomie chez les adolescentes usagères du Centre de santé des femmes de la Mauricie. L'autonomie désigne, ici, un pouvoir sur soi et un contrôle sur sa réalité (Corbeil et al., 1983; Dagenais, 1985 ; Théoret et al., 1986). L'intégration de ce concept se concentre sur le processus contraceptif parce que les adolescentes s'y présentent pour avoir la pilule anticonceptionnelle. En raison du cadre conceptuel du mouvement féministe pour la santé des femmes, pratiquer une contraception et poser des gestes préventifs et curatifs contre les MTS, sont alors considérés comme des signes d'autonomie et des comportements d'autosanté (Collectif de Boston, 1971 ; De Koninck et al. , 1983 ; Vandelac, 1983 ; Pelletier, 1985 ; Collectif du Centre de Santé de Trois-Rivières, 1986 ; Regroupement des Centres de Santé, 1986).

Les féministes définissent l'autosanté dans leurs luttes politiques, leurs services alternatifs autogérés et leurs discussions sur la sexualité (Hite, 1976; Kerr, 1977; L'Heureux, 1979) comme une réaction à la société sexiste/patriarcale où le privé est vu comme politique (Melucci, 1983 ; Van Den Bergh et Cooper, 1987). L'autonomie acquise à travers la dimension santé/sexualité peut donc être extrapolée aux autres sphères de vie (Regroupement des Centres de Santé, 1986). C'est à partir de ce recadrage vers une analyse sociale de situations individuelles, que fut introduit, dans la grille d'entrevue, ce thème sur le partenaire (s'il y avait lieu), ou sinon, sur la relation avec les garçons.

\section{Méthodologie}

En plus de ce cadre conceptuel féministe, cette étude se distingue par la méthodologie qualitative de cueillette et d'analyse des données d'entrevue. Nous désirions donner parole à des adolescentes sur divers thèmes connexes au processus contraceptif : la contraception, la sexualité, les maladies transmises sexuellement et, ici, les relations hommes - femmes.

Les données de cette recherche ont été recueillies auprès d'un échantillon typique de 10 adolescentes, représentatif de la populationmère de 68 usagères (Langlois et Gagnon, 1973). Cet échantillon respecte la représentation minimale de $10 \%$ exigée pour une étude qualitative (Gagnon, 1985). À partir des indications de la problématique, il est sélectionné selon les critères suivants : l'âge (entre 15 et 18 ans), le 


\section{TABLEAU 1}

\section{Extrait de la grille d'entrevue sur le thème : relations hommes - femmes}

Tu m'as déjà dit quelle était ta vie amoureuse actuellement.

Si tu as un chum régulier, quelle implication a-t-il à propos de la contraception et des MTS ?

Quelle implication devrait-il avoir?

Si tu n'as pas de chum stable, quelle devrait être l'implication des gars à propos de la contraception et des MTS?

Comment vois-tu la relation avec les gars ? La vie amoureuse?

Penses-tu à d'autres choses sur la vie amoureuse, sur tes relations avec les gars, avec les hommes, ou sur ta sexualité afin de me permettre de mieux te comprendre?

temps de fréquentation au Centre et la classe sociale. La classe sociale de l'adolescente est déterminée par le statut social du "chef de famille", la scolarité et l'occupation du parent qui vit avec l'enfant, ou celui dont le revenu est le plus élevé lorsque les parents cohabitent et travaillent.

La cueillette des propos se réalise à l'aide d'une grille à questions ouvertes, dans le cadre d'une entrevue individuelle, semi-dirigée, d'une heure environ. Le tableau 1 présente les questions qui concernent spécifiquement le thème touché par cet article.

Nous avons constaté, lors de ces entrevues, une forte implication devant les sujets proposés. Les adolescentes parlaient avec une grande ouverture de leur intimité affective, nous fournissaient des détails sur les amis, sur les jeux de séduction ou sur leurs opinions concernant les garçons. Le contenu de ces entrevues est étudié selon la méthode d'analyse de contenu où l'attention est portée sur la représentation, c'est-à-dire sur le sens des mots. "L'analyse de contenu apparaît comme un ensemble de techniques d'analyse de communications utilisant des procédures systématiques et objectives de description de contenu des messages. 》 (Bardin, $1977:$ 38).

Dans les tableaux subséquents descriptifs du contenu discursif, nous indiquerons toute nouvelle donnée qui décrira avec plus de précisions l'idée à l'étude ; il s'agit de la technique de l'absence et de la présence. Par ailleurs, pour faciliter la compréhension de ces entrevues, le contenu a été classé selon un système de catégories et de souscatégories. Nous avons réalisé ce que René L'Écuyer (1986) appelle l'établissement des catégories mixtes, soit des catégories préexistantes 
jumelées aux catégories à induire. Dans un premier temps, des subdivisions ont été fixées à partir de la définition opérationnelle du terme " autonomie " basée sur la liste des comportements correspondant à ce concept intégré selon les féministes et la pratique du Centre. L'intégration du concept d'autonomie signifie en effet que des comportements précis sont manifestés dans la description du vécu des adolescentes, que des mots sont présents dans leur langage, que des idées se dégagent de leur discours. Dans un second temps, nous avons reclassé les premières subdivisions afin de mieux les adapter au langage. Des catégories élargies et homogènes sont alors apparues. C'est ainsi que pour ce thème (comme pour les autres), les sous-catégories définitives sont donc regroupées sous les titres : sentiments, appuis et autonomie.

La catégorie "sentiments" regroupe toute émotion ressentie par l'adolescente face aux garçons. Pour faciliter la compréhension, nous avons induit un regroupement intermédiaire entre cette catégorie et la sous-catégorie "émotion ressentie». Les adolescentes distinguent, en effet, la qualité relationnelle et affective avec leur partenaire, de celle de leurs contacts, soit avec les autres gars et soit surtout avec les groupes de garçons. Les appuis et les obstacles correspondent à l'apport des réseaux au développement harmonieux des relations avec les gars. En dernier lieu, nous identifierons les moyens que les adolescentes se donnent pour vivre une certaine distance par rapport à leur couple, une certaine liberté vis-à-vis de leur partenaire, et ainsi une autonomie. Nous noterons aussi ce qui gêne, selon elles, cette autonomie, ce qui les empêche de vivre un pouvoir sur elles-mêmes et sur leur vécu affectif.

En outre, chaque sous-catégorie sera qualifiée par des phrases où des mots clé expriment l'idée de l'interviewée (unité d'enregistrement) sur le thème (unité de contexte). Ces unités d'enregistrement seront analysées selon les règles de la co-occurrence associative énoncées par Laurence Bardin (1977) où " la co-occurrence est la présence simultanée de deux ou plusieurs unités d'enregistrement dans une unité de contexte " (p. 112). Plus précisément, les associations identifiées dans les tableaux ci-dessous regroupent les qualificatifs en accord avec le thème, les oppositions étant des obstacles au développement harmonieux des relations hommes/femmes.

Chaque tableau est suivi d'une discussion dans le respect des deux niveaux d'abstraction (Bardin, 1977 ; Laperrière, 1982; Horth, 1986). Le premier niveau (analyse) correspond directement à la situation substantive ; l'utilisation par les filles de certains termes et mots devient le reflet de leur vision de leur relation avec les garçons. Le second niveau d'abstraction (interprétation) questionne leurs propos en fonction principalement de l'intégration de l'autonomie. Comme il pourra être observé, le langage sur ce thème précis a été peu relevé dans la revue de 
la littérature, sauf dans certaines réflexions des féministes sur l'oppression des femmes et ce, lors de leur discussion sur l'intervention féministe.

\section{Sentiments vécus par les adolescentes dans leurs relations hommes/femmes}

Pour ces adolescentes, leur couple est, tout d'abord, un lieu de dialogue où il se dit " des affaires intéressantes ». Ces échanges résolvent les tensions interpersonnelles. Trois d'entre elles disent avoir des problèmes de communication ; elles précisent les vivre avec une intensité moindre, dans leur vie de couple, grâce à la compréhension de leur partenaire. Nous constatons qu'il s'agit des trois adolescentes qui vivent des difficultés familiales.

"En communication, disons moi je ne suis pas toujours correcte avec, disons gentille. Puis il reste impassible, toujours fin."

"Il me pose des questions style: "Pourquoi t'es avec moi ?" ou je ne sais pas, j'ai de la misère à le dire. Parce qu'en plus c'est un gars. Mais il le comprend, avec ce que je vis avec mon beau-père. "

Les idées ici mentionnées se perçoivent aussi lorsque les interviewées parlent de la prise de décision conjointe pour une contraception, et de la participation de leur partenaire à cette contraception. N'est-ce pas là notamment l'intégration de valeurs et de principes mis de l'avant par la psychologie moderne, dans le domaine de l'harmonie conjugale? Cette communication ne permet-elle pas de créer une intimité, et partant, une distance par rapport aux parents?

Les jeunes filles associent également à leur vie de couple, une entraide qu'elles disent provenir du dialogue. À l'étude de leurs propos, nous constatons qu'elles y expriment davantage l'aide reçue de leur ami, qu'une entraide réciproque, partagée et égalitaire. Pouvons-nous interpréter une telle différence, comme une relation à tendance dominant-dominée, où une seule personne a vraiment besoin d'aide? Les inhabiletés supplémentaires que l'adolescente subit, pour s'intégrer à la société, en raison de sa socialisation déficiente, ne sont-elles pas confirmées dans sa vie privée, par cette inéquité d'entraide ? En effet, le partenaire, au lieu de l'ouvrir à une autonomie grâce à une analyse critique (Sturdivant, 1980; Corbeil et al., 1983; Russell, 1984) ne la maintiendra-t-il pas dans une dépendance affective, avec ce réconfort émotif?

À travers ce contact va s'installer un respect de la part du partenaire. Ce respect se vit davantage lorsqu'ils "sont ensemble», dans leur 


\section{TABLEAU 2}

\section{Sentiments dans les relations hommes/femmes}

\begin{tabular}{|c|c|}
\hline Sentiments & Quelques propos des adolescentes \\
\hline \multicolumn{2}{|l|}{ Associations : } \\
\hline Dialogue & $\begin{array}{l}\text { "On jase tout le temps des affaires qui sont } \\
\text { intéressantes" } \\
\text { "ÂA place de se chicaner on va discuter, s'il y a } \\
\text { de quoi qui ne marche pas, quand même } \\
\text { qu'on serait trois heures assis (...) ça s'ar- } \\
\text { range." }\end{array}$ \\
\hline Entraide & $\begin{array}{l}\text { "Il me pousse pour mes études" } \\
\text { "Quand j’ai un problème, j'en parle à mon } \\
\text { chum, qu'il le veuille ou non et il va m'écou- } \\
\text { ter" } \\
\text { "C'est lui qui ramasse les morceaux" } \\
\text { "Je le prends, pas comme un psychologue là, } \\
\text { mais" } \\
\text { "l'avais un problème de drogue pis lui aussi. } \\
\text { On s'est dit que peut-être ensemble on serait } \\
\text { capable de régler nos problèmes" }\end{array}$ \\
\hline Respect & $\begin{array}{l}\text { "Les gars qui vont sortir habituellement avec } \\
\text { une fille vont la respecter" } \\
\text { "Tout seul, il se tranquillise, il te respecte" } \\
\text { "Il sait que bon, avant d'être menstruée, faut } \\
\text { pas qu'il me brasse" }\end{array}$ \\
\hline Affection & $\begin{array}{l}\text { "Cen'est pas a priori un romantique mais il est } \\
\text { attentif, j'ai l'affection que je veux" } \\
\text { "Je vois dans ses yeux qu'il me comprend" } \\
\text { "Quand on était juste nous deux, il n'était pas } \\
\text { pareil. Il était plus démonstratif" } \\
\text { "Je ne veux pas lui parler de ses sentiments } \\
\text { parce que j'ai peur qu'il reparte, mais il en a" }\end{array}$ \\
\hline Confiance & $\begin{array}{l}\text { "J'ai jamais été ouverte comme ça (..) j'ai } \\
\text { jamais dit à personne que je pleurais le soir } \\
\text { avant de m'endormir" } \\
\text { "ll va garder ça pour lui " }\end{array}$ \\
\hline Responsabilité & $\begin{array}{l}\text { "Je me conditionne un peu à moins crier, à } \\
\text { moins rusher, pis à moins m'enfermer dans } \\
\text { mon coin (...) à être plus, à l'accepter. Pis lui } \\
\text { aussi, fait ça" } \\
\text { "ll faut que tu saches si le gars, il va faire un } \\
\text { bon père, s'il prend ses responsabilités" }\end{array}$ \\
\hline
\end{tabular}




\begin{tabular}{|c|c|}
\hline Oppositions : & \\
\hline Déviance & $\begin{array}{l}\text { "Ils avaient un party, c'est la grosse défonce" } \\
\text { "Cette gang-là, on consommait de la drogue" } \\
\text { "Pas sérieux, pas sérieux du tout. Ça revire } \\
\text { toujours en jokes, en niaisages" }\end{array}$ \\
\hline Irrespect & $\begin{array}{l}\text { "Ils sont pareils. En gang ils déraisonnent là } \\
\text { dessus, sur le sexe. (...) Ils passent leurs com- } \\
\text { mentaires, lui comme les autres" } \\
\text { "À ses chums, il dit: "Ah ma blonde elle suce } \\
\text { bien"." } \\
\text { "lls peuvent te prendre pour des poupées" }\end{array}$ \\
\hline Égocentrisme & $\begin{array}{l}\text { "Quand c'est juste pour une soirée, il pense } \\
\text { juste à lui " } \\
\text { "Lui il s'en fout quand même qu'elle n'aime } \\
\text { pas ça, il ne la reverra pas le lendemain" }\end{array}$ \\
\hline Vantardise & $\begin{array}{l}\text { "Eux autres, c'est comme si ça les remontait de } \\
\text { nous écraser" } \\
\text { "lls se vantent quand les blondes ne sont pas } \\
\text { là. Ils disent : avec elles, je suis de même." } \\
\text { "Je vais te dire que les gars de mon âge, vont se } \\
\text { traîner un condom dans leur portefeuille pour } \\
\text { se valoriser face à leurs chums" } \\
\text { "C'est parce que ça c'est de la jalousie. Ils } \\
\text { disaient tout le temps: Ah elle je l'ai eue, je } \\
\text { l'aurai encore" }\end{array}$ \\
\hline Oppression & $\begin{array}{l}\text { "La fille c'est une guidoune; le gars c'est un } \\
\text { champion" } \\
\text { "Le gars lui il n'aura pas de reproches. (...) ils } \\
\text { ne sont pas jugés (...) elle va être jugée elle. } \\
\text { Mais elle ne l’a pas fait toute seule" } \\
\text { "'en ai déjà eu un qui avait une réputation de } \\
\text { gars qui fait tout le tour de la ville. Je me } \\
\text { sentais mal juste à cause de ça " } \\
\text { "ll se faisait du placotage, des choses pas vraies } \\
\text { (...) ta réputation, elle se fait. T'as l'air intelli- } \\
\text { gente. T'as de la misère à te faire des amies } \\
\text { après (...), ça te bloque un peu partout à cause } \\
\text { de ça " }\end{array}$ \\
\hline
\end{tabular}

intimité ; il en sera de même pour l'affection. Ces propos laissent filtrer les oppositions ultérieures. Le partenaire a des comportements appréciés dans la vie privée, mais il s'en abstient en public. Les adolescentes y vivent alors une affection qu'elles ne retrouvent pas chez d'autres garçons, ainsi qu'une qualité, une intensité, une densité, qu'elles ne 
rencontrent pas ailleurs. Nous observons que certains gars ont de la difficulté à exprimer clairement et verbalement leurs émotions, leurs désirs et leurs sensations. Ce malaise se dégage, particulièrement, des propos des filles qui disent éprouver elles-mêmes des difficultés de communication. Nous percevons aussi beaucoup de tendresse de leur part, et par les qualificatifs employés, une certaine idéalisation de leur partenaire. Il va, finalement, s'établir dans leur vie de couple une confiance mutuelle, un sens des responsabilités et un certain partage pour la qualité du climat. Elles associent donc des émotions positives, agréables, épanouies et épanouissantes, à une vie à deux idyllique, à un partenaire qui satisfait leurs besoins.

Ce partenaire présente le portrait du garçon idéal, lorsqu'il est comparé aux autres gars, et surtout à leurs groupes. Elles les voient oppresseurs; elles opposent ainsi le couple au groupe mixte, mais surtout masculin. Elles associent en effet le groupe à la déviance. Elles y sentent un irrespect à leur égard, et ce, surtout dans les moqueries des gars sur la sexualité. Tel que sous-entendu précédemment, le partenaire devient " comme les autres " lorsqu'il est en groupe, avec ses " chums".

Elles renchérissent en attribuant aux garçons un égocentrisme et une vantardise. Elles ressentent ces situations comme une oppression. Ainsi, elles distinguent une fois de plus le couple du groupe, et différencient nettement l'ami d'un soir du partenaire stable. À notre avis, le même garçon peut possiblement recevoir les deux qualificatifs, successivement, de deux filles différentes. L'égocentrisme sera notable au niveau de la sexualité et sur le plan affectif ; la vantardise se perçoit en terme de domination, surtout en ce qui a trait à la sexualité. L'oppression sera pour sa part associée spécialement à des préjugés, à des jugements de valeur dénigrant l'activité sexuelle féminine, valorisant ou excusant la sexualité masculine.

En définitive, nous constatons l'ampleur des qualificatifs utilisés. Pour le partenaire, ces qualificatifs sont teintés de tendresse ; mais pour le groupe, les autres gars, l'ami d'un soir, ils sont noircis d'oppression. Dans nos choix méthodologiques et épistémologiques, nous avons établi notre alliance avec les interviewées, ne mettant pas en doute leurs propos et les croyant, tels qu'ils étaient exprimés. Malgré ces considérations, nous questionnons l'intensité des qualificatifs utilisés pour décrire les sentiments vécus. L'ami est-il si affectueux ? Le groupe est-il si dénigrant et si déviant, sans qu'elles n'en portent aucune responsabilité? Ne sont-elles pas complices (de façon tacite tout au moins) par leur silence, par l'absence de leur contre-discours, à l'oppression du groupe mixte? N'excusent-elles pas les comportements de leur partenaire par l'influence du groupe? 
Nous interprétons ainsi ces propos : le garçon présente sûrement une différence de comportement, dans son intimité, et avec ses amis. Le groupe doit, de fait, être porteur de préjugés et de réputations. Ces jugements négatifs peuvent aussi être véhiculés par d'autres filles de leur âge. Nous induisons finalement, de ces propos, une dépendance des filles à l'égard de leur ami, puisque ce dernier représente un portrait idyllique du conjoint, de l'amour, du support affectif et des relations hommes-femmes. Nous nous interrogeons, de plus, sur leur difficulté de s'intégrer, ou plutôt d'avoir du pouvoir dans des groupes mixtes; en effet, elles se questionnent peu sur leurs propres comportements de séduction. Par contre, une contrainte inhérente à cette recherche est ici mise en lumière : nous n'avons pas le point de vue masculin s'appliquant au même sujet à savoir les relations hommes-femmes. Finalement, si nous comparons ces dires et ces situations à l'analyse féministe de la société sexiste/patriarcale, nous y interprétons, dans ces situations individuelles et privées, la reproduction de cette hiérarchie sociale, en microcosme; en effet, ces relations affectives, sous le regard d'une analyse sexiste, nous apparaissent globalement en faveur du privilège masculin, et en fonction de la socialisation déficiente des filles. Bref, le privé y est politique (Melucci, 1983 ; Van Den Bergh et Cooper, 1987).

C'est ainsi qu'à partir de ce constat, l'utilisation du terme "harmonieux" pour qualifier des relations hommes/femmes, fait à la fois référence à une vision féministe qui insiste sur l'épanouissement individuel, androgyne, sans une domination de l'un sur l'autre, et au langage des adolescentes qui recherchent le dialogue, le respect mutuel et l'affection.

\section{Appuis au développement des relations hommes-femmes harmonieuses}

L'appui du partenaire, pour qu'un couple vive des relations harmonieuses, est associé à l'âge de l'ami et à sa maturité : sa stabilité comportementale et sa capacité de communication. Les filles interviewées fréquentent toutes, sauf une, des garçons de plus de 20 ans. Ceux-ci vont à l'université, poursuivent d'autres études terminales, ou sont à la recherche d'un emploi. Aucune n'est en amour avec un garçon de son âge, fréquentant la même classe. Cette maturité liée à l'âge, elles l'intègrent aussi à la capacité chez un couple de résoudre ses conflits. Elles se reconnaissent une maturité acquise, grâce au passage de 14 à 18 ans, même si, en général, elles font porter aux garçons cette nécessité 


\section{TABLEAU 3}

\section{Appuis au développement des relations hommes/femmes harmonieuses}

\begin{tabular}{|c|c|}
\hline Appuis & Quelques propos des adolescentes \\
\hline \multicolumn{2}{|l|}{ Associations : } \\
\hline Maturité liée à l'âge & $\begin{array}{l}\text { "Les gars restent plus eux-mêmes tout le temps" } \\
\text { "Ils sont capables de jaser" } \\
\text { "Là, on devient plus mature on est capable de } \\
\text { résoudre nos problèmes" }\end{array}$ \\
\hline Confiance des parents & $\begin{array}{l}\text { "Tout en demandant des permissions, pis que } \\
\text { mes parents sachent où je suis (...) je peux faire } \\
\text { (...) ce quej'ai envie de faire. D'ailleurs ils peuvent } \\
\text { me faire confiance" } \\
\text { "Ça fait } 5 \text { ans que je sors avec, elle a confiance" }\end{array}$ \\
\hline Support des pères & $\begin{array}{l}\text { "Mon père m'a juste mise en garde (...) ça va } \\
\text { t'empêcher, ça te bloque" } \\
\text { "C'est le fun, on se comprend super bien, on se } \\
\text { parle beaucoup" } \\
\text { "C'est ce que lui, de la façon dont il me percevait, } \\
\text { pis comme moi je le ressentais, c'était pas tout à } \\
\text { fait pareil. Mais y'avait raison" }\end{array}$ \\
\hline \multicolumn{2}{|l|}{ Oppositions (obstacles) : } \\
\hline $\begin{array}{l}\text { Immaturité liée } \\
\text { au jeune âge }\end{array}$ & $\begin{array}{l}\text { "À } 15 \text { ans, c'est tout nébuleux ce que tu vis" } \\
\text { "À } 14 \text { ans, ils niaisent tout le temps. Les gars eux } \\
\text { autres, ils vieillissent de } 14 \text { à } 17 \text { ans" }\end{array}$ \\
\hline $\begin{array}{l}\text { Manque d'information } \\
\text { en psychologie }\end{array}$ & $\begin{array}{l}\text { "Les gars par rapport aux filles comment ils se } \\
\text { sentent, pis la première fois qu'ils font l'amour. } \\
\text { C'est ça qu'on a le goût d'entendre" } \\
\text { "Un peu des relations humaines, l'importance de } \\
\text { l'amour (...) espèces d'éducation de l'amour (...) } \\
\text { L'importance de la communication dansle couple, } \\
\text { parce qu'il y a des choses que tu peux } \\
\text { apprendre" }\end{array}$ \\
\hline Peur des mères & $\begin{array}{l}\text { "Ma mère avait peur (...) Ma mère m'empêchait } \\
\text { de sortir avec mon ami (...) je la détestais (...) "T'es } \\
\text { une petite courailleuse" " } \\
\text { "Si j'avais pas d'ami, je ne pourrais pas sortir } \\
\text { parce que j'en rencontrerais un" }\end{array}$ \\
\hline
\end{tabular}


d'acquérir une maturité. L'immaturité liée au jeune âge s'oppose, donc, à des relations affectives harmonieuses.

Nous interprétons l'importance à accorder à l'âge dans la problématique adolescente. Vivre une vie amoureuse, tout comme avoir une contraception, est une situation plus épanouie à 17 qu'à 15 ans. La vision globale de la sexualité (Frappier, 1980 ; Tessier, 1985; Wilkins, 1985 ; Desrosiers, 1986) le confirme à travers le développement psychologique de l'adolescente, puisque cette maturation apporte un support à de tels comportements. Mais nous décelons tout de même une certaine magie et un romantisme, lorsque les jeunes filles associent la résolution des problèmes conjugaux presque uniquement à cette maturation de l'âge, et surtout lorsqu'elles sous-entendent la poursuite ultérieure d'un tel cheminement.

En outre, l'école apparaît comme un appui potentiel. Elles lui demandent de discuter, non seulement de méthodes contraceptives, mais aussi des informations sur les relations hommes-femmes, sur la sexualité et sur la psychologie de la communication. Ce manque de connaissance nuit à leurs relations amoureuses. Les adolescentes s'interrogent sur leurs (quelques) tensions avec leur ami et les garçons. Une faille, selon nous, semble se dissimuler à leurs yeux, dans les explications qu'elles fournissent pour comprendre cette réalité, que ce soit l'impact néfaste du groupe de "chums " ou l'obstacle de l'âge. Le programme de "Formation personnelle et sociale", actuellement en implantation, répondrait, selon le ministère de l'Éducation, à ces préoccupations (Décary, 1987; Desaulniers, 1987 ; Pelletier, 1987).

L'ouverture des parents, identifiée comme essentielle à une contraception, est ici qualifiée, selon les propos des filles concernant l'apport des parents dans l'apprentissage de la vie amoureuse, par un seul mot : la confiance. Elles aspirent à ce qu'on leur fasse confiance, elles apprécient quand cela a lieu et s'en considèrent dignes. Ces marques d'estime parentale sont fonction de leurs propres comportements, et aussi, des agirs du partenaire.

À notre avis, une différence de langage semble départager la situation des deux adolescentes des milieux les plus aisés et de celles de classes plus défavorisées. Les premières insistent plus sur l'encadrement supportant leur liberté d'action, sur la souplesse facilitatrice de l'éducation reçue, et sur l'appui de leur père. Ces derniers discutent avec elles de leur relation amoureuse, pour un cheminement vers l'autonomie. Par ailleurs, leur mère appuie activement leur contraception par une collaboration directe. Quant aux deux autres, elles mettent l'accent sur les peurs de leur mère, et, sur la confiance que celles-ci acquièrent auprès du partenaire. Elles qualifient leur mère d'«autoritaire" et de "questionneuse "; elles considèrent que ce contexte s'oppose à une vie 
amoureuse et entrave leur épanouissement. La problématique de la contraception (Frappier, 1982) identifie d'ailleurs un risque plus élevé de grossesses indésirées dans des milieux économiquement plus défavorisés.

Devant ces insistances différentes, surgit une double observation. Une empreinte du discours féministe et du langage de la psychologie moderne se fait plus visible dans les classes aisées, influençant ainsi, entre autres, le comportement des pères. Pour leur part, les adolescentes de milieu défavorisé sont-elles exposées à un environnement social plus dangereux, ou plus propice aux risques de grossesses indésirées? Cette constatation apporterait un jalon d'explication aux réactions plus réticentes des mères.

\section{Autonomie à vivre dans les relations hommes-femmes}

Les filles voient leurs amies comme un système supportant leur autonomie. D'une part, elles partagent avec elles leur vécu avec leur ami. Elles discutent des plaisirs, des tensions et des tristesses; elles tentent de trouver des alternatives à certains comportements et situations. D'autre part, réaliser des loisirs avec leurs amies fournit des occasions pour se libérer du couple et explorer leur autonomie. Il est possible de constater que les adolescentes qui donnent une plus grande emphase affective à l'amitié sont celles qui vivent des tensions familiales, s'attribuent des problèmes de communication et identifient chez leur partenaire des difficultés à exprimer ses émotions. L'amitié apparaît, alors, comme le réseau d'aide privilégié et l'appui à l'équilibre personnel.

De plus, les adolescentes proposent des amitiés hommes-femmes, comme un moyen pour apprendre à être bien avec des garçons, à mieux les connaître et à vivre leur propre autonomie. Deux d'entre elles associent cette amitié à des garçons au " caractère plus féminin ». Nous interprétons que de telles attitudes peuvent leur apparaître moins menaçantes. Deux autres précisent que les garçons adoptent des comportements différents ; ils sont " pas pareils qu'avec les autres filles ", ou ils agissent pour que l'adolescente "garde sa confiance ". Celles qui sont issues des milieux les plus favorisés, insistent davantage sur la possibilité et l'importance de telles amitiés et sur la complicité qu'elles réussissent à créer. $Y$ a-t-il un lien entre l'ouverture vécue avec le père, et cette capacité de vivre des relations amicales et égalitaires avec des garçons? L'expérimentation avec le père ne devient-elle pas une source de confiance en soi et une capacité de contact comme le proposent 


\section{TABLEAU 4}

\section{Autonomie à vivre dans les relations hommes-femmes}

\begin{tabular}{|c|c|}
\hline Autonomie & Quelques propos des adolescentes \\
\hline \multicolumn{2}{|l|}{ Associations : } \\
\hline $\begin{array}{l}\text { Support du } \\
\text { groupe d'amies }\end{array}$ & $\begin{array}{l}\text { "Avec Isabelle, je peux dire n'importe quoi" } \\
\text { "Â ma chum de fille, je jase de mon chum" } \\
\text { "C'est le fun sortir avec mes amies de filles" }\end{array}$ \\
\hline $\begin{array}{l}\text { Apprentissage par une } \\
\text { amitié homme-femme }\end{array}$ & $\begin{array}{l}\text { "Ils me prennent pour une grande amie à eux } \\
\text { autres. Envers moi, ils ne réagissent pas pareil } \\
\text { qu'avec les autres filles" } \\
\text { "J'ai (...) un grand ami gars, il est pas mal féminin, } \\
\text { ça fait que ça aide sûrement " } \\
\text { "Les gars, ça ne veut pas dire que c'est des mania- } \\
\text { ques. T'sais y savent que j'ai confiance en eux } \\
\text { autres. Ça fait qu'ils veulent que je garde ma } \\
\text { confiance" }\end{array}$ \\
\hline $\begin{array}{l}\text { Contrôle sur la } \\
\text { relation affective }\end{array}$ & $\begin{array}{l}\text { "Moi pas le premier soir" } \\
\text { "Des fois, j'aurais le goût de me laisser aller } \\
\text { mais...j'ai peur à demain (...) Je ne veux pas, je me } \\
\text { suis fait jamais niaiser" } \\
\text { "Je me suis rendue indépendante envers mon } \\
\text { chum." } \\
\text { "Lève-toi pis fais tes choses toi-même" } \\
\text { "On ne se voit pas trop la semaine, je suis aux } \\
\text { études" } \\
\text { "J'ai essayé de lui dire que j'aimerais ça le voir } \\
\text { moins souvent." }\end{array}$ \\
\hline \multicolumn{2}{|l|}{ Oppositions (obstacles) : } \\
\hline Possessivité & $\begin{array}{l}\text { "Il est ben jaloux (...) Il me retient. (...) Il a ben de } \\
\text { la misère à comprendre que je veux sortir avec } \\
\text { mon amie de fille" }\end{array}$ \\
\hline $\begin{array}{l}\text { Peur de perdre la } \\
\text { relation affective }\end{array}$ & $\begin{array}{l}\text { "J'ai peur de le perdre. Je l'aime mais... j'aimerais } \\
\text { pas ça lui faire de la peine, mais j'ai encore besoin } \\
\text { de liberté. (...) II ne comprendrait pas, j'ai trop } \\
\text { attendu pour lui dire aussi clairement " }\end{array}$ \\
\hline
\end{tabular}


Appleton (1986) et Olivier (1986) ? De plus, les garçons qu'elles côtoient, probablement aussi de classe aisée, sont-ils donc différents eux aussi, en raison du modelage sur un père plus ouvert et plus respectueux?

De plus, les jeunes filles désignent le contrôle sur la relation affective comme favorable à une autonomie. À l'instar des contraceptifs qui sont un instrument de régulation de la relation sexuelle, l'affirmation de soi et le respect de son corps apparaissent ici comme des attitudes et des moyens à privilégier. Nous constatons donc, à travers cette démarche d'autonomie, l'intégration d'attitudes associées à l'intervention féministe (Corbeil et al., 1983) : l'affirmation de soi, la fierté de son corps et une certaine autodétermination.

L'une d'elles déplore ne pas pouvoir prendre sa place malgré ses efforts; d'ailleurs, elle oppose son autonomie à sa peur de perdre son ami, qui « ne comprendrait pas » son "besoin de liberté ", alors qu'elle l'aime. C'est ainsi que l'autonomie peut être minimisée par cette possessivité et par la jalousie du partenaire. Puisque seulement trois d'entre elles mentionnent de telles difficultés, est-ce que ce serait là un signe qu'une évolution puisse avoir lieu chez ces adolescents? Les garçons seraient-ils moins possessifs que les hommes adultes, et les filles moins dépendantes que leurs aînées? Ces dix jeunes filles seraient-elles plutôt au début d'un processus de conscientisation, ayant une intuition devant les moyens à prendre, une conscience relative de leur démarche d'autonomie, et une réflexion limitée sur l'oppression vécue dans leur couple?

\section{Constatations globales}

Les adolescentes ont une image très positive de leur vie de couple, en même temps qu'un sentiment d'oppression avec des groupes de garçons. Leur partenaire, par contre, devient comme les autres en public, alors qu'il est affectueux en privé. Nous nous demandons si leur image de la réalité, idéalisée du partenaire, idyllique de la vie amoureuse, et noircie des autres gars, n'accroît pas une possibilité de dépendance vis-à-vis de leur ami, et de négation des difficultés de couple.

Nous voyons aussi des signes d'autonomie et des désirs de liberté chez ces filles. Mais nous ne pouvons guère affirmer leur contrôle sur leur réalité affective, ainsi qu'elles peuvent le réussir sur des gestes concrets comme la contraception. En effet, il faut tenir compte de leurs besoins émotifs, leur insécurité affective et leur socialisation déficiente. Elles ont de plus à transiger avec leur partenaire quant à sa maturité, son 
ouverture, son écoute, sa disponibilité, sa compréhension et sa socialisation. Cette situation amène une perspective sociétale où le privé est signe de la société sexiste.

Par ailleurs, les parents peuvent contribuer par leur confiance ou nuire par leur peur au développement harmonieux des relations amoureuses de leur adolescente. L'école est un réseau dont les adolescentes attendent des informations supportant leur développement. Le groupe d'amies prend place lors de la discussion concernant l'autonomie dans le cadre de relations hommes-femmes; il devient ainsi un système d'aide grâce auquel il est possible de vivre leur autonomie aux côtés de leur ami. 


\section{TABLEAU 5}

\section{Résumé des catégories concernant la perception des relations hommes-femmes}

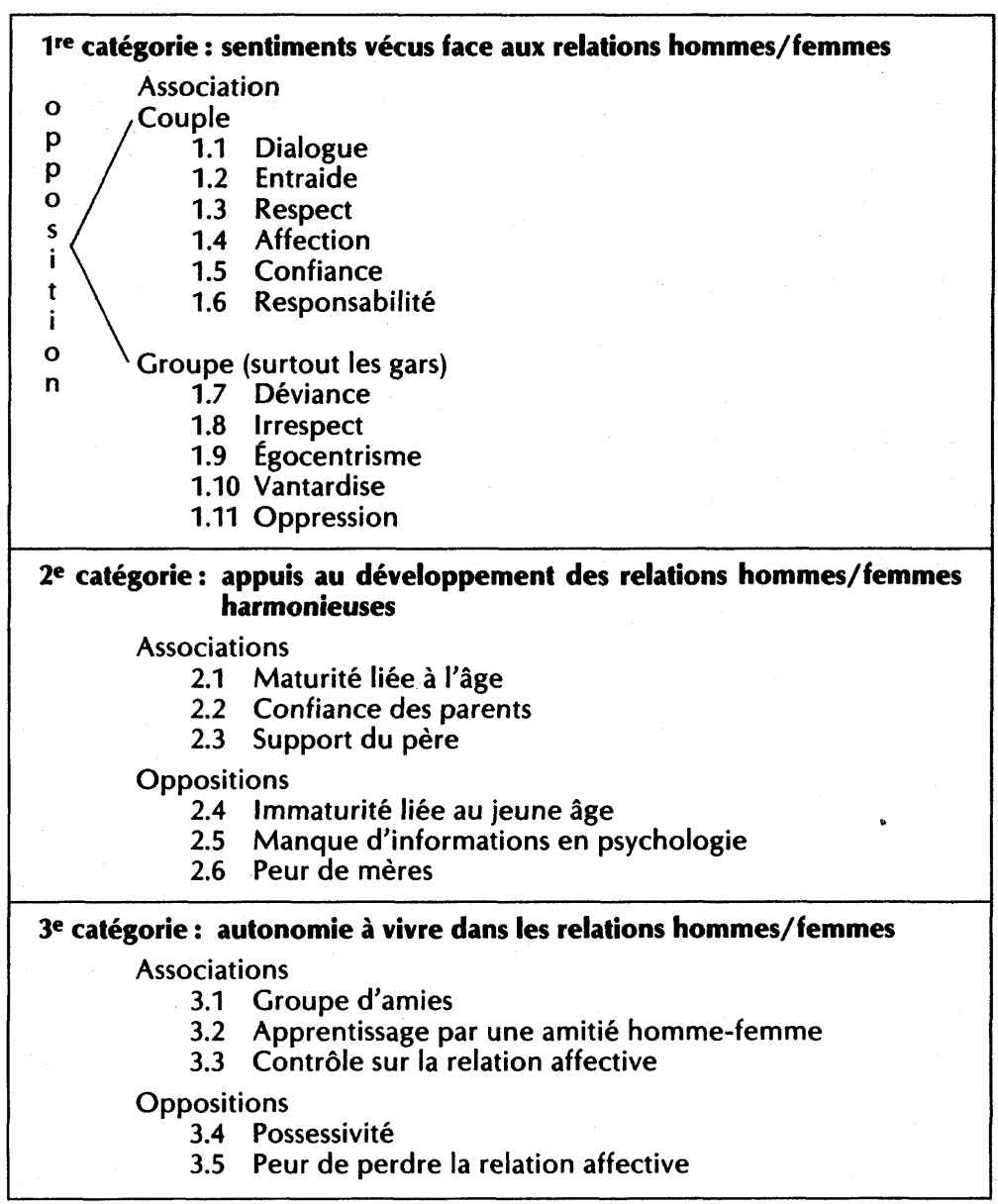




\section{Conclusion}

En guise de conclusion, nous aimerions élargir les propos de cet article en y ajoutant les constatations principales de l'étude de référence. En raison de la méthodologie qualitative, ainsi qu'en raison de l'échantillon restreint et privilégié, nous ne nous hasardons pas à des généralisations. Nous ouvrons plutôt des pistes de réflexion pour des intervenant(e)s auprès d'adolescentes. Cette recherche peut donc être qualifiée d'inductive, d'exploratoire et de descriptive d'une clientèle précise et d'un lieu spécifique d'intervention féministe ; puis, elle apparaît comme un support à des interrogations ultérieures.

En définitive, les usagères de 15-18 ans intègrent davantage l'autonomie à des gestes concrets et à des situations précises comme :

- la contraception;

- une démarche d'autosanté vis-à-vis des M.T.S. ;

- des aspirations professionnelles leur permettant d'accéder à une indépendance financière.

Son intégration est moindre, par rapport à une compréhension et à des situations plus globales touchant :

- les relations hommes/femmes, notamment dans un processus contraceptif ;

- la sexualité ;

- les aspirations familiales, entre autres l'organisation des tâches dans leur future famille.

Trois principaux facteurs influencent leurs gestes autonomes : l'âge, les préjugés sociaux et la stabilité affective avec leur partenaire. En effet, les gestes reliés au processus contraceptif sont plus fréquents et posés de façon plus épanouie à 17 ans qu'à 15 ans. C'est ainsi que les adolescentes peuvent venir au Centre de santé, avoir une contraception, vivre une sexualité satisfaisante, et partager une relation harmonieuse avec un ami qui aura 20 ans. Les préjugés sociaux, qui peuvent être perçus chez les divers réseaux les entourant, diminuent au fur et à mesure que l'adolescente vieillit et acquière de la maturité. Ces jugements de valeur portent sur sa possible instabilité affective. Ceux qui proviennent du milieu familial, spécialement de la mère, ont un effet marquant sur leurs gestes autonomes entraînant parfois la confidentialité à propos des services gynécologiques et la clandestinité de la vie sexuelle.

En outre, les adolescentes du présent échantillon ont une vie amoureuse relativement stable. Elles vivent leurs gestes autonomes dans le cadre d'une stabilité affective, définie comme étant l'unicité d'un partenaire; mais elles se réfèrent alors à une courte période de 
temps. Par contre, nous n'avons pas pu déceler d'influence significative due à l'appartenance à une classe sociale dans cet échantillon; les adolescentes issues de milieux très favorisés semblent tout de même avoir une socialisation plus alternative, moins limitative, eu égard entre autres à leur choix de carrière, et en raison notamment de la collaboration plus active du père à leur éducation et à l'organisation familiale.

Quatre agents de socialisation exercent une influence sur leur définition de l'autonomie, et surtout sur son intégration dans des comportements indicateurs :

1. La famille:

- le modelage sur la mère et sur l'organisation familiale

- l'influence due à la participation du père

2. L'école :

- un lieu de diffusion d'informations

- des préjugés sociaux portés surtout par les pairs

- un support important des amies intimes

3. Les mass-médias :

- un lieu de diffusion d'informations sur les M.T.S.

- un véhicule de valeurs et de stéréotypes sexistes

4. Le mouvement social des femmes:

- le modelage sur les intervenantes du Centre de santé des femmes de la Mauricie

- une perception négative des féministes, spécialement des plus radicales, malgré une intégration de certaines notions concernant la condition des femmes.

Bref, les adolescentes vivent d'importants enjeux engageant leur avenir de femmes épanouies et autonomes. Leurs amours sont un lieu et un enjeu central où elles sont confrontées à des plaisirs, des tensions et des questions. Elles n'espèrent que l'écoute et le support d'adultes pour aller plus loin, d'hommes et de femmes, d'intervenants et de parents. Leurs paroles nous ont-elles convaincu(e)s?

\section{Notes}

* Nous tenons à remercier mesdames Francine Ouellet et Geneviève Martin, respectivement directrice et co-directrice de recherche, pour leur support tout au long du mémoire dont cet article fait mention. Nous désirons aussi souligner l'excellente collaboration du Collectif du Centre de santé des femmes de la Mauricie, et l'apport notable, à chaque étape de cette étude, de mesdames Diane Carle et Clo Pratte. 


\section{Références bibliographiques}

APPLETON, William, "The role of the father ", dans : Le temps d'y voir ; Conférence internationale sur la situation des filles (1985), Montréal, Guérin, 1986 : 9-19.

BARDIN, Laurence, L'analyse de contenu, Paris, Presses universitaires de France, 1977.

Beaulieu, Ginette, "Alerte rouge aux M.T.S.", Québec Science, vol. 24, no 5, $1986: 16-24$.

Beaulieu, Ginette, "Lutte aux M.T.S. : la campagne des régions", Santé Société, vol. 9, n० 2, $1987: 30-34$.

Charbonneau, Louise, Ghislaine Fortin et Monique Tessier, "La clinique des jeunes Saint-Denis, six ans plus tard ", Santé mentale au Québec, vol. IX, no 2, $1984: 74-82$.

Collectif de Boston pour la santé des femmes, Notre corps, nous mêmes, adapté en 1977 de l'américain par un collectif de femmes, Paris, Éditions Albin Michel, 1971.

Collectif du Centre de santé des femmes de la Mauricie, Représentation écrite à la Commission d'enquête sur les services de santé et les services sociaux, Trois-Rivières, Centre de santé des femmes de la Mauricie, 1986 (inédit).

Corbelt, Christine et al., L'intervention féministe; l'alternative des femmes au sexisme en thérapie, Montréal, Éditions coopératives Albert Saint-Martin, 1983.

Dagenals, Huguette, "Les femmes et le pouvoir dans le domaine de la santé", dans: Les femmes et la santé, Chicoutimi, Éditions Gaëtan Morin, 1985 : 107-118.

DECARY, Yves, "Un pas de plus vers la connaissance de soi : l'éducation à la sexualité à l'école», Conférence présentée au Colloque sur l'éducation sexuelle, organisé par le Département de santé communautaire (D.S.C.) du Centre hospitalier Sainte-Marie, Trois-Rivières, 1987.

De Koninck, Maria, Francine Saillant et Lise Dunnigan, Essai sur la santé des femmes, Québec, Conseil du statut de la femme, 1983.

DESAULNIERS, Marie-Paule, "La place des valeurs en éducation sexuelle ", Conférence présentée au Colloque sur l'éducation sexuelle organisé par le D.S.C. du Centre hospitalier Sainte-Marie, Trois-Rivières.

Desrosiers, Marie, "La sexualité dans le contexte normal de l'adolescence des années 80 ", Intervention, no 73, $1986: 24-30$.

Drolet, Marie, "L'intégration de la définition féministe du concept d'autonomie chez les adolescentes usagères du Centre de Santé des femmes de la Mauricie ", Mémoire déposé à l'École des gradués de l'Université Laval en décembre 1987 pour l'obtention d'une maîtrise en service social, 1987 (inédit).

FrAPPIER, Jean-Yves, Adolescence et sexualité, Conférence présentée en 1985 au Colloque sur Sexualité et Adolescence, organisé par le D.S.C. du Centre hospitalier Sainte-Marie, Trois-Rivières, 1980, (inédit).

FrapPIER, Jean-Yves, "Prise en charge de l'adolescente qui fait usage de contraception ", texte remis au Colloque Sexualité et Adolescence, organisé par le 
D.S.C. du Centre hospitalier Sainte-Marie, Trois-Rivières, 1982 et publié dans: Médecine et hygiène du 12 mars 1982.

GAGNON, Damien, " 2 millions pour la lutte au SIDA", Le Soleil, 25 août 1987, pp. A-1, A-2, A-3.

GAGNON, Nicole, Méthode quantitative de l'analyse de contenu, Québec, Laboratoire de recherches sociologiques, Département de sociologie, Université Laval, 1985.

GeRMAIN, Georges-Hébert, "La nouvelle guerre mondiale : l'effet SIDA ", L'Actualité, vol. 12, n० 5, $1987: 32-42$.

HITE, Share, Le rapport Hite, traduit de l'américain par Théo Carlin, Paris, Éditions Robert Laffont, 1976.

HORTH, Raynald, L'approche qualitative comme méthodologie de recherche en Science de l'éducation, Pointe-au-Père, Les Éditions de la Mer, 1986.

KANTROWITZ, Barbara et al., "Kids and contraception", Newsweek, 16 février $1987: 54-65$.

KERR, Carmen, Le sexe au féminin, traduit de l'anglais par Danielle Simpson, Montréal, Les éditions de l'Homme, 1977.

LANGLOIS, Simon et Nicole GAGNON, "Les techniques de l'échantillonnage ", notes du cours Analyse des données II, Département de sociologie, Université Laval, 1973, (inédit).

LAPERRIÈRE, Anne, «Pour une construction empirique de la théorie: la nouvelle école de Chicago ", Sociologie et société, vol. XIV, n 1, 31-41.

L'ÉCUYER, René, "L'analyse de contenu: notion étapes", dans La recherche qualitative; résurgence et convergences, Chicoutimi, Groupe de recherche et d'intervention régionales de l'Université du Québec à Chicoutimi, 1986 : 65-89.

L'Heureux, Christine, L'orgasme au féminin, Montréal, Les éditions Univers Inc., 1979.

MetuCCI, Alberto, "Mouvements sociaux, mouvements post-politiques ", Revue internationale d'action communautaire, vol. 10/50, automne $1983: 13-31$.

Ouvier, Christiane, "Jeune fille et identification féminine, dans: Le temps d'y voir ; Conférence internationale sur la situation des filles (1985), Montréal ; Guérin, 1986, p. 29.

OUELLET-DUBE, Francine, "Le niveau social et l'utilisation des services à l'enfance", mémoire de maîtrise présenté à l'École de service social, Université Laval, 1976 (inédit).

Pelletier, Francine, "Le Centre de Santé de Montréal, 10 ans plus tard, La vie en rose, no 27 , juin 1985 : 14-15.

Pelletier, Francine, "La sexualité fait ses classes", Châtelaine, vol. 28, n० 11, $1987: 65-70$.

Regroupement des centres de santé de femmes du Québec, Représentation écrite présentée à la Commission d'enquête sur les services de santé et les services sociaux, Hull, Regroupement des Centres de santé, 1986 (inédit).

RusselL, Mary Nomme, Skills in Counselling Women; The Feminist Approach, Springfield, Charles C. Thomas publisher, 1984. 
STEBEN, Marc, "Les maladies transmises sexuellement ", dans : Le temps d'y voir ; conférence internationale sur la situation des filles (1985), Montréal, Guérin, 1986a : 195-201.

STEBEN, Marc, "Les maladies transmises sexuellement : l'épidémie catastrophique », dans: Jeunesse et sexualité; actes du colloque, Longueuil, Éditions IRIS, 1986b : 519-527.

StURDIVANT, Susan, Les femmes et la psychothérapie; une philosophie féministe du traitement, traduit de l'américain, Bruxelles, Pierre Mardaga éditeur, 1980.

TESSIER, Monique, "Adolescence et sexualité : les enjeux de la prévention ", Santé mentale au Québec, vol. 9. n², 1984 : 64-73.

TESSIER, Monique, Sexualité et prévention; d'abord l'affaire des jeunes, Montréal; Bureau de consultation jeunesse, 1985.

TESSIER, Monique, "Sexualité adolescente et prévention : des choix s'imposent ", dans : Jeunesse et sexualité; actes du colloque, Longueuil, Éditions IRIS, 1986 : 529-547.

ThEOREt, Manon et al., "Le plaisir ça s'apprend, le pouvoir aussi », dans : Le temps d'y voir; conférence internationale sur la situation des filles (1985), Montréal, Guérin, 1986 : 301-331.

VANDELAC, Louise, "Femmes, santé, notre pouvoir ", dans : Nous, notre santé, nos pouvoirs, Montréal, Éditions coopératives Albert Saint-Martin, 1983 : 183-197.

VAN Den BerGH, Nan et Lynn COOPER, "Introduction ", dans : Féminist visions for Social Work, Silver Spring, National Association of Social Workers, 1986 : 1-28.

WIIKINS, Jean, " La sexualité durant l'adolescence : phénomène pluridimensionnel », dans: Médecine de l'adolescence: une médecine spécifique, Montréal, Hôpital Sainte-Justine, 1985 : 172-182. 